\title{
Calculation of the Conformational Entropy of Polysilane ${ }^{\dagger}$
}

\author{
Mengbo LUO and Jianmin $\mathrm{XU}^{*}$ \\ Department of Physics, Hangzhou University, Hangzhou, \\ 310028, People's Republic of China \\ (Received July 4, 1995)
}

\begin{abstract}
Exact enumeration method is used to calculate the conformational entropy of polysilane chain, submitted to short-range interaction and long-range interaction, with an off-lattice RIS model. The conformational entropy is in proportion to chain length at any temperature (from $50 \mathrm{~K}$ to $400 \mathrm{~K}$ ). It varies with temperature, can be expressed as the function of temperature. And we find the second-order transition temperature $\left(T_{2}\right)$ of polysilane is about $65 \mathrm{~K}$
\end{abstract}

KEY WORDS Exact Enumeration Method/Conformational Entropy/Polysilane

Conformations of polymer chains depend largely on conformational entropy which is associated with the order of the system. Calculation of the conformational entropy is an important and hard work. The entropy of a polymer chain at $\theta$-state in a dilute solution (the chain submitted only to short-range interaction) can be calculated with the method of Flory's matrix multiplication. ${ }^{1}$ But this method will be ineffective when long-range interaction prevails among the chain segments. $^{2}$

There are several methods for calculating the conformational entropy of polymer chain perturbed by long-range interaction. Exact enumeration ${ }^{2-4}$ of all the possible conformations can calculate the entropy of such system, but it is only able to deal with short chain (10-20 segments) due to the tremendous number of conformations for long chain. Meirovitch's scanning method can treat relatively long chain. ${ }^{5.6}$ More recently, Collet and Premilat ${ }^{7}$ proposed a method for calculating the conformational entropy of much longer polymer chains based on the mean probabilities obtained from Monte Carlo simulation and applied it successfully on polyethylene chain.

Among all these methods which are used to calculate the entropy of perturbed chain, including those not mentioned here, the exact enumeration can provide most precise results, others can only provide approximate results. So, it is widely used in polymer science, ${ }^{2-4,8-10}$ which results are often treated as an important check of other methods. ${ }^{5}$ In the present work, we propose to calculate the conformational entropy of the polysilane chain with the exact enumeration method. The calculation is performed on an off-lattice three rotational isomeric state (RIS) model of polysilane. The polymer chain was submitted not only to short-range interaction (RIS), but also to long-range interaction (excluded volume in the work) in a manner similar to that proposed by Jianmin et al. $^{2}$

\section{METHOD OF CALCULATION}

The skeleton atoms of the polysilane chain are all the

\footnotetext{
${ }^{\dagger}$ This work was supported by the Natural Science Foundation of Zhejiang Province, China.

** To whom all correspondence should be addressed.
}

silicon, the bond length of the $\mathrm{Si}-\mathrm{Si}$ is fixed as $2.34 \AA$, the bond angle of the $\mathrm{Si}-\mathrm{Si}-\mathrm{Si}$ is $109.4^{\circ}$. The primary statistical weight matrix is ${ }^{11}$

$$
U=\left[\begin{array}{ccc}
1 & \sigma & \sigma \\
1 & \sigma \psi & \sigma \omega \\
1 & \sigma \omega & \sigma \psi
\end{array}\right]
$$

where

$$
\begin{aligned}
& \sigma=\exp \left(-E_{\sigma} / k T\right) \\
& \psi=\exp \left(-E_{\psi} / k T\right) \\
& \omega=\exp \left(-E_{\omega} / k T\right)
\end{aligned}
$$

and $E_{\sigma}=-0.278 \mathrm{kcal} \mathrm{mol}^{-1}, \quad E_{\psi}=-0.240 \mathrm{kcal} \mathrm{mol}^{-1}$, $E_{\omega}=0.387 \mathrm{kcal} \mathrm{mol}^{-1}, k$ is the Boltzmann constant.

The long-range interaction between non-neighbor atoms $i$ and $j$ is represented by a hard sphere potential which represents an excluded volume ${ }^{2,7}$

$$
E_{i j}= \begin{cases}\infty, & r_{i j}<r_{\mathrm{c}} \\ 0, & r_{i j} \geqslant r_{\mathrm{c}}\end{cases}
$$

$r_{i j}$ is the distance between two atoms, and $|i-j|$ must greater than 4. Simply, we only consider the skeleten atoms $\mathrm{Si}$, chain side atoms $\mathrm{H}$ are neglected, ${ }^{12} r_{\mathrm{c}}$ is chosen as the Van der Waals radii of $\mathrm{Si}-\mathrm{Si}$ in this paper, so $r_{\mathrm{c}}$ is $4.10 \AA .{ }^{11}$ Since the statistical properties of an isolated chain (chain in a dilute solution) are independent of its external coordinates, they only depend on the internal rotation of bonds of the chain, so conformations of a chain with $N$ bonds are determined by the internal rotation of $N-2$ bonds, thus the conformational entropy of the chain depends on the rotation of $N-2$ bonds. ${ }^{1}$ Therefore, the first two bonds are fixed in our calculation, the next $N-2$ bonds are generated with an off-lattice model, each has three possible orientation $\left(t, g^{+}, g^{-}\right)$. The successive two rotational angles are determined by ${ }^{11}$

\begin{tabular}{ccccc} 
& $t t$ & $t g$ & $g t$ & $g^{ \pm} g^{ \pm}$ \\
\hline$\phi_{i}, \phi_{i+1}$ & $\left(0^{\circ}, 0^{\circ}\right)$ & $\left(0^{\circ}, \pm 125^{\circ}\right)$ & $\left( \pm 125^{\circ}, 0^{\circ}\right)$ & $\left( \pm 125^{\circ}, \pm 125^{\circ}\right)$ \\
\hline & $g^{ \pm} g^{\mp}$ & & & \\
\hline
\end{tabular}

$\phi_{i}, \phi_{i+1}\left( \pm 120^{\circ}, \mp 120^{\circ}\right)$

All the possible conformations of the polysilane chain of $\mathrm{N}$ bonds are generated. Then the partition function 
can be calculated $\mathrm{as}^{2,13}$

$$
Z=\sum_{i} \exp \left(-E_{i} / k T\right)
$$

where $E_{i}$ is the energy of conformation $i$ and the summation is over all the possible conformations. $T$ is the temperature, $k$ is the Boltzmann constant as in eq 1 .

The mean energy of the polymer chain is:

$$
\langle E\rangle=Z^{-1} \cdot \sum_{i} E_{i} \cdot \exp \left(-E_{i} / k T\right)
$$

Then the conformational entropy per mol of the chain is:

$$
S=R \cdot \ln Z+\langle E\rangle / T
$$

here $R=N_{0} k, N_{0}$ is Avogadro constant, $R=1.987$ cal $\mathrm{K}^{-1} \mathrm{~mol}^{-1}$.

In this paper, the conformation entropies $S$ at different temperature are calculated, the temperatures range from $50 \mathrm{~K}$ to $400 \mathrm{~K}$. Since it takes a great deal of CPU time of a computer, the chain length is limitted to 17 bonds.

\section{RESULTS AND DISCUSSION}

The conformational entropies of short polysilane chain submitted to short-range interaction (RIS) and longrange interaction (hard sphere) at temperature from $50 \mathrm{~K}$ to $400 \mathrm{~K}$ are calculated, while the chain length $N$ is from 7 up to 17 . The number of all possible conformations of such chain is listed in Table I.

The conformational entropy per mol $S$ versus $\mathrm{N}-2$ is shown in Figure 1 for the temperature from $50 \mathrm{~K}$ to $400 \mathrm{~K}$. Results show that the conformational entropy is a linear function of $N-2$ at any temperature, give a relationship similar to that obtained for much longer chain using Monte Carlo method by Collet $^{7}$ :

$$
S=a(N-2)+b
$$

The reason we use $N-2$ instead of chain length $N$ here is that the internal rotations of $N-2$ bonds contribute to the conformational entropy.

$a$ and $b$ in eq 6 are dependent on the temperature, the values of $a$ and $b$ at different temperature are listed in Table II. $a$ and $b$ can be expressed as the function of temperature. Using the least square method, we get $a$ and $b$ which are good for the temperature from $50 \mathrm{~K}$ to $400 \mathrm{~K}$,

$$
\begin{aligned}
a= & 2.380 \times 10^{-2}-0.6745 \tau+7.229 \tau^{2}-4.448 \tau^{3} \\
& -7.602 \tau^{4}+6.748 \tau^{5} \\
b= & 0.6980+0.5737 \tau+4.624 \tau^{2}-29.20 \tau^{3} \\
& +42.53 \tau^{4}-19.21 \tau^{5}
\end{aligned}
$$

where $\tau$ has a relationship to temperature $T, \tau=$ $\exp (\varepsilon / k T) . \varepsilon$ is minus, the magnitude of $\varepsilon$ is arbitrary, we choose $\varepsilon=E_{\sigma}=-0.278 \mathrm{kcal} \mathrm{mol}^{-1}$ here. One merit of choosing $\tau$ instead of $T$ in eq 7 is that $a$ and $b$ will converge when temperature tends infinity. Also, the mean square deviation of $a$ and $b$ are largely decreased when we use $\tau$, especially at high temperature. The value of $a$ and $b$ under $50 \mathrm{~K}$ or above $400 \mathrm{~K}$ can be estimated from the tendency of them near $50 \mathrm{~K}$ or $400 \mathrm{~K}$.

The linear relations of $S$ versus $N-2$ are so good that Polym. J., Vol. 28, No. 3, 1996
Table I. The number of all possible configurations of the polysilane chain submitted to short-range interaction and long-range interaction ${ }^{\mathrm{a}}$

\begin{tabular}{ccccccc}
\hline$N$ & 7 & 8 & 9 & 10 & 11 & 12 \\
\hline$\Omega$ & 143 & 363 & 937 & 2405 & 6137 & 15701 \\
\hline$N$ & 13 & 14 & 15 & 16 & 17 & \\
\hline$\Omega$ & 40115 & 102401 & 261297 & 666125 & 1697653 &
\end{tabular}

${ }^{a} N$ is the chain length (defined as the number of bonds of the chain), $\Omega$ is the number of all the possible configurations.

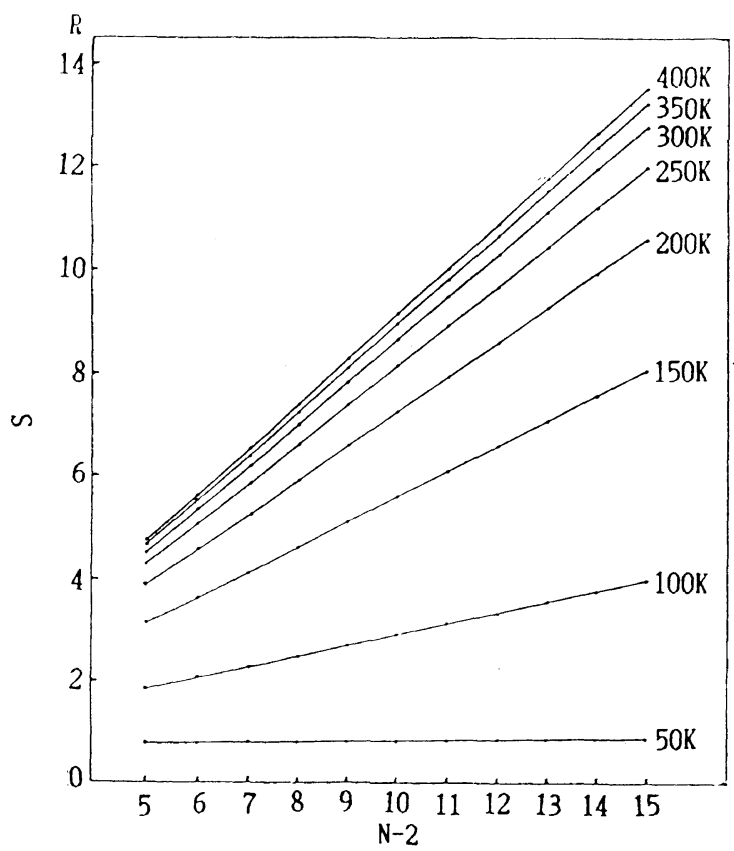

Figure 1. The plots of the configuration entropy per mol $S$ versus $N-2$ at different temperature, $R=1.987 \mathrm{cal} \mathrm{K}^{-1} \mathrm{~mol}^{-1}$.

Table II. Values of $a$ and $b$ at different temperature

\begin{tabular}{clc}
\hline \multicolumn{1}{c}{$T$} & \multicolumn{1}{c}{$a$} & $b$ \\
\hline $50 \mathrm{~K}$ & $8.432 \times 10^{-3}$ & 0.7441 \\
$100 \mathrm{~K}$ & 0.2088 & 0.8225 \\
$150 \mathrm{~K}$ & 0.4880 & 0.6989 \\
$200 \mathrm{~K}$ & 0.6689 & 0.5535 \\
$250 \mathrm{~K}$ & 0.7694 & 0.4509 \\
$300 \mathrm{~K}$ & 0.8257 & 0.3889 \\
$350 \mathrm{~K}$ & 0.8590 & 0.3518 \\
$400 \mathrm{~K}$ & 0.8799 & 0.3290
\end{tabular}

it seems reasonable to consider that the relations are still valid for long chain. Let $S_{\mathrm{m}}$ stand for the entropy per bond per mol of an infinity chain, then

$$
\begin{gathered}
S_{\mathrm{m}}=\lim _{N \rightarrow \infty} S /(N-2) \\
=a
\end{gathered}
$$

In Figure 2, we show $S /(N-2)$ versus temperature $T$ for chain length $N=7,12$, and $17, S_{\mathrm{m}}$ versus $T$ is also shown. It indicates that $S /(N-2)$ decreases as $N$ increase, and converges rapidly when $N$ increases. Since the internal rotation of bonds contributes the conformational entropy, when the chain length increases, the space near the bond is gradually occupied by other bonds, the 


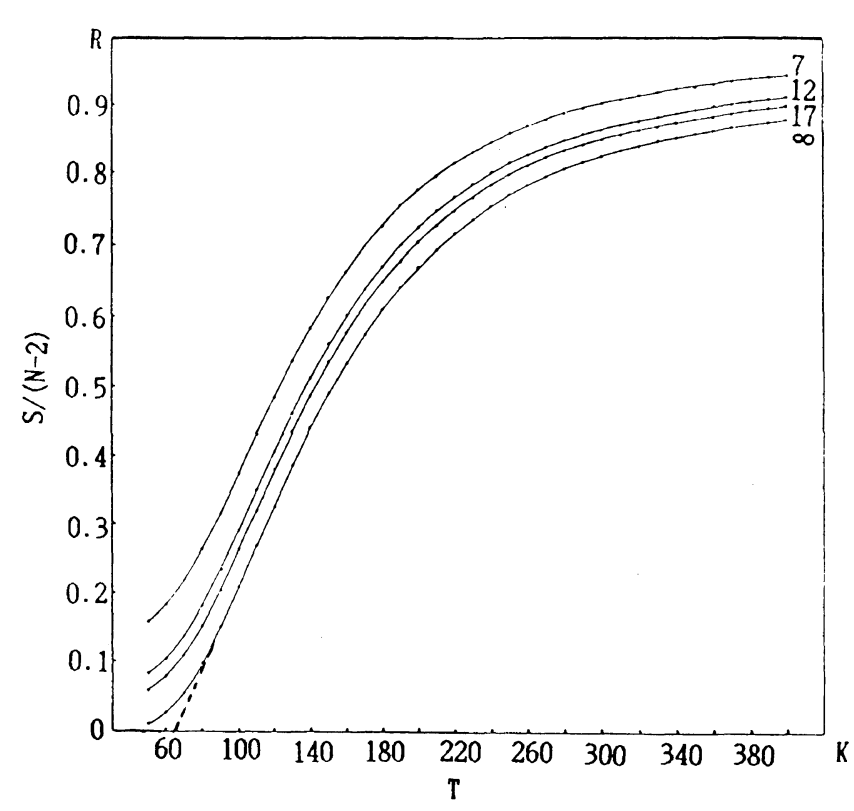

Figure 2. The plots of the configuration entropy per internal rotation angle $S /(N-2)$ versus the temperature, for chain length $N=7,12,17$, and $N=\infty, R=1.987 \mathrm{cal} \mathrm{K}^{-1} \mathrm{~mol}^{-1}$

rotation of the bond is obstructed by degrees, so the entropy $S /(N-2)$ decreases steadily.

Figure 2 also shows that the conformational entropy decreases as the temperature decreases. This agrees with Gibbs-DiMarzio's explanation about the relation between conformation and temperature. ${ }^{14}$ They thought that the conformations of polymer chains rearranged when the temperature decreased. At high temperature, the chains didn't prefer to any special conformations, but at low temperature, the chains began to prefer some low energy conformations. When the temperature reached $T_{2}$ (second-order transition temperature), the chains preferred to the lowest energy conformations, therefore the conformational entropy became zero. Because of the limitation of the computer's precision, the error of calculation will be gradually large when one reduces the temperature, for some of the factors $\exp \left(-E_{i} / k T\right)$ in eq 3 and 4 become very small or very large at very low temperature. Therefore the calculation of the entropy of very low temperature becomes difficult and even impossible. Thus, $T_{2}$ must be determined by extrapolating from high temperature.

The curve of $S_{\mathrm{m}}$ versus $T$ is almost straight in the region between $80 \mathrm{~K}$ and $140 \mathrm{~K}$ where the entropy $S_{\mathrm{m}}$ varies rapidliest. Extrapolating to the lower temperature from this linear region, one will find that the crossover temperature between $S_{\mathrm{m}}=0$ and $S_{\mathrm{m}}$ for high temperature range is about $65 \mathrm{~K} . S_{\mathrm{o}}$, the $T_{2}$ of polysilane chain which submitted to short-range interaction and an excluded volume is about $65 \mathrm{~K}$.

Exact enumeration method has been used in attempt to calculate the conformational entropy of polysilane chain submitted to short-range interaction and an excluded volume. The conformational entropy appears proportional to chain length at any temperature. It varies with temperature, and show that the second-order transition temperature of polysilane in dilute solution is about $65 \mathrm{~K}$, but we can't check it for lack of experimental value.

\section{REFERENCES}

1. P. J. Flory, "Statistical Mechanics of Chain Molecules," Wiley, New York, N.Y., 1969.

2. X. Jianmin, S. Xubing, and Z. Zhiping, Eur. Polym. J., 25, 601 (1989).

3. M. F. Sykes, A. J. Guttman, M. G. Watts, and P. D. Roberts, J. Phys., A5, 653 (1972).

4. D. C. Rapaport, J. Phys., A9, 1521 (1976)

5. H. Meirovitch, Macromolecules, 16, 249 (1983).

6. H. Meirovitch, Macromolecules, 18, 563 (1985).

7. O. Collet and S. Premilat, Macromolecules, 26, 6076 (1993).

8. J. L. Martin, M. F. Sykes, and F. T. Hioe, J. Chem. Phys., 46 3478 (1967).

9. C. Domb and F. T. Hioe, J. Chem. Phys., 51, 1915 (1969).

10. M. A. Moore, J. Phys., A10, 305 (1977).

11. W. J. Welsh, L. DeBolt, and J. E. Mark, Macromolecules, 19, 2978 (1986).

12. F. T. Wall, S. Winder, and P. J. Gans, J. Chem. Phys., 37, 1461 (1962).

13. J. Mazer and F. L. McCrackin, J. Chem. Phys., 49, 648 (1968).

14. J. H. Gibbs and E. A. DiMarzio, J. Chem. Phys., 28, 373 (1958). 